\title{
On the DMT Optimality of the Rotate-and-Forward Scheme in a Two-Hop MIMO Relay Channel
}

\author{
Ramtin Pedarsani, Olivier Lévêque, Sheng Yang
}

\begin{abstract}
The rotate-and-forward scheme was introduced in (Yang-Belfiore, 2010) to recover spatial diversity in multi-hop MIMO relay networks. It was shown that this scheme achieves the optimal diversity-multiplexing (DMT) trade-off in a two-hop relay network, with two antennas at the relay node. In this paper, it is shown that the scheme is DMT optimal for arbitrary number of antennas at the source, relay, and destination node.
\end{abstract}

\section{INTRODUCTION}

The gain of using multiple antennas for setting up communication over a wireless medium has been widely acknowledged in the literature, starting with the seminal works [1], [2]. For point-to-point channels, the performance of multiple antenna systems is quite well understood by now. In particular, the optimal tradeoff between reliability and rate (also known as diversitymultiplexing tradeoff or DMT) of such systems at high SNR was analyzed in detail in [3].

In recent years, there has been a surge of interest in cooperative diversity techniques, where spatial diversity is exploited with distributed relay antennas. Many different schemes have been proposed to improve the diversity of the channel (see, e.g., [5], [6], [7], [8], [9], [10], [11], [12], [16], [13], [14], [15] and references therein). Essentially, these schemes can be divided into two categories, namely, linear and nonlinear relaying schemes. Based on message decoding (e.g., decodeand-forward) or signal compression (e.g., compressand-forward) or a mixture of both at the relays, nonlinear relaying schemes are "intelligent" and can usually outperform the "dumb" linear relaying schemes (e.g., amplify-and-forward) where relays only forward linear combinations of individual observations. It is worth noting that a nonlinear scheme called quantize-andforward, recently proposed in [14], has been shown to achieve any rate within a constant number of bits to the

R. Pedarsani and O. Lévêque are with the School of Computer and Communication Sciences, EPF Lausanne, Switzerland, \{ramtin.pedarsani, olivier. leveque\}@epfl.ch

$\mathrm{S}$. Yang is with the Telecommunications Department, Supélec, Gif sur Yvette, France sheng.yangesupelec.fr capacity of multi-hop relay networks and thus attains the optimal DMT of such networks.

On the other hand, linear relaying schemes are appealing for their low complexity. More importantly, it has been shown that they can be DMT optimal in some settings [10], [17]. It is worth mentioning that an interesting linear scheme, called the flip-and-forward scheme, was proposed in [16], and in [18], it was shown that it is DMT optimal when there are two antennas at the relay. Another interesting linear relaying scheme, called the rotate-and-forward scheme, was proposed in [17], and it was shown to be DMT optimal when the relay node is equipped with two antennas. However, the proof for the optimality of this scheme in a more general setting was still open. In this paper, we prove the DMT optimality of the rotate-and-forward scheme in a two-hop MIMO relay network with an arbitrary number of antennas at the source, relay and destination.

The rest of the paper is organized as follows. The system model and the main theorem on the DMT optimality of the rotate-and-forward scheme are presented in Section II. From there, we build the necessary tools for the proof of the main theorem. The strategy for the proof is as follows. We first obtain an asymptotic behavior of the mutual information, which involves the determinant of a combination of random matrices. Then, we obtain a lower bound on the determinant which can be expressed as a simple function of independent random variables with known distributions. This lower bound gives an upper bound on the outage probability which in turn yields a lower bound on the diversity order. From the joint distribution of the random variables involved in the lower bound on the mutual information, we compute the corresponding diversity order, following the methodology of [3], and show that this lower bound matches the optimal DMT found by the cutset bound. In Section III, we first show a corresponding lower bound on the DMT of the classical MIMO channel, which turns out to be the key for proving the DMT optimality of the rotateand-forward scheme. In Section IV, we complete the 


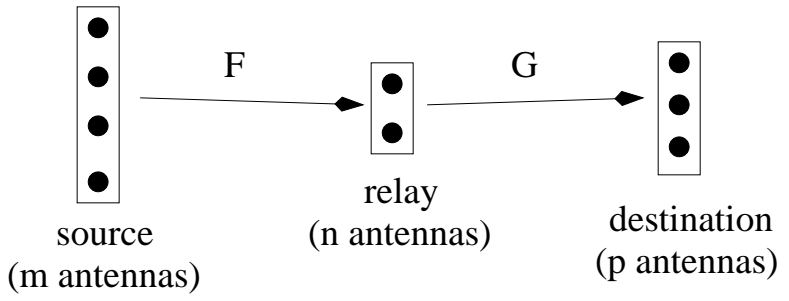

Fig. 1: Two-hop MIMO relay channel.

lower bound on the rotate-and-forward scheme and compute the corresponding DMT. Finally, we conclude in Section V and details of proofs are provided in the Appendix.

\section{System Model}

We consider a two-hop relay channel with $m$ antennas at the source, $p$ antennas at the destination and $n$ antennas at the relay ${ }^{1}$. We assume that there is no direct link between the source and the destination, and that the relay is full-duplex. Let $F$ denote the $n \times m$ channel matrix between the source and the relay, and $G$ denote the $p \times n$ channel matrix between the relay and the destination (see Figure 1). Furthermore, the channel state is assumed to be known at the receivers, but not at the transmitters.

The considered relaying scheme can be described by the following signal model:

$$
\begin{aligned}
& y_{R}[t]=F x[t]+z_{R}[t], \\
& x_{R}[t]=\Delta[t] y_{R}[t-1], \\
& y_{D}[t]=G x_{R}[t]+z_{D}[t],
\end{aligned}
$$

where $x \in \mathbb{C}^{m \times 1}, x_{R} \in \mathbb{C}^{n \times 1}, y_{R} \in \mathbb{C}^{n \times 1}$ and $y_{D} \in \mathbb{C}^{p \times 1}$ are the transmitted signal from the source, transmitted signal from the relays, received signal at the relays, and received signal at the destination, respectively; $z_{R} \in \mathbb{C}^{n \times 1}$ and $z_{D} \in \mathbb{C}^{p \times 1}$ are the additive white Gaussian noise with unit covariance at the relay and the destination, respectively. $\Delta[t] \in \mathbb{C}^{n \times n}$ is a diagonal matrix.

We are interested in the high SNR behavior of this system. In this work, we will focus on a distributed relaying scheme, i.e. no information on the message or channel state information (CSI) is exchanged between the relays.

In order to evaluate the performance of the relaying scheme, we use the diversity-multiplexing tradeoff

\footnotetext{
${ }^{1}$ Since we do not consider joint processing of different antennas, the results apply to an arbitrary number of relays and $n$ generally refers to the total number of antennas within the layer of relays.
}

(DMT) introduced in [3] for MIMO systems. A relaying scheme is said to achieve multiplexing gain $r$ and diversity gain $d(r)$ if

$$
d(r)=\lim _{\mathrm{SNR} \rightarrow \infty}-\frac{\log \left(\mathbb{P}_{\text {out }}(r \log \mathrm{SNR})\right)}{\log \mathrm{SNR}},
$$

where $\mathbb{P}_{\text {out }}(r \log \mathrm{SNR})$ denotes the outage probability, that is, the probability that the mutual information between the source and the destination is lower than the target rate $R=r \log$ SNR.

In the rotate-and-forward scheme proposed in [17], a linear relaying scheme is performed, according to a fixed sequence of diagonal matrices. Let us define a set of $L$ equally spaced angles in $[0,2 \pi)$ and the corresponding set of complex rotations as follows

$$
\begin{aligned}
& \mathcal{A}_{L} \triangleq\left\{0, \frac{2 \pi}{L}, \ldots, \frac{2(L-1) \pi}{L}\right\}, \\
& \mathcal{R}_{L} \triangleq\left\{e^{j \phi} \mid \phi \in \mathcal{A}_{L}\right\} .
\end{aligned}
$$

Definition 1: A sequence of diagonal matrices $(\Delta[1], \ldots, \Delta[T])$ with $T=L^{n}$ is said to be a distributed rotation sequence (DRS) if

1) $\Delta[t]=\operatorname{diag}\left(\xi_{1}[t], \ldots, \xi_{n}[t]\right)$ with $\xi_{i}[t] \in \mathcal{R}_{L}$,

2) $\Delta[t] \neq \Delta\left[t^{\prime}\right], \forall t \neq t^{\prime}$.

A fixed DRS is used by the relays to create time-varying channels. A codeword $X \triangleq(x[1], \ldots, x[T]) \in \mathbb{C}^{m \times T}$ spanning over $T$ time symbols is transmitted by the source with. At time $t$, each relay transmits a rotated version of what it received at instant $t-1$. The rotation used by the relay $i$ is $\xi_{i}[t]$, the $i^{t h}$ element of the vector $\xi[t]$. Therefore, the equivalent channel in the high SNR regime can be formulated as ${ }^{2}$

$$
y_{R}[t+1]=G \Delta[t] F x[t]+G \Delta[t] z_{R}[t]+z_{D}[t+1],
$$

for $t=1, \cdots, T$. Hence, the transmitted codeword $X$ goes through an equivalent time-varying fading channel with channel matrix $G \Delta[t] F$ and noise covariance $\Sigma_{z}=I+G G^{*}$ (where $A^{*}$ denotes the complexconjugate transpose of $A$ ).

In the high SNR regime, we can ignore the noise covariance for the DMT analysis. Consequently, the mutual information between the source and the destination can be approximated as

$$
\begin{aligned}
& I_{T}(\mathrm{SNR}) \\
& \quad=\frac{1}{T} \sum_{t=1}^{T} \log \operatorname{det}\left(I_{n}+\operatorname{SNR} \Delta[t] F F^{*} \Delta[t]^{*} G^{*} G\right)
\end{aligned}
$$

\footnotetext{
${ }^{2}$ Due to the power constraint, a normalization factor $\sqrt{\frac{\mathrm{SNR}}{\mathrm{SNR}+1}}$
} is applied. This factor is ignored in the high SNR regime. 
and

$$
\mathbb{P}_{\text {out }}(r \log \mathrm{SNR}) \doteq \mathbb{P}\left(I_{T}(\mathrm{SNR})<r \log \mathrm{SNR}\right),
$$

where $\doteq$ denotes asymptotic exponential equality, as defined in [3]. The corresponding DMT was studied in [17] in the particular case of 2 antennas at the relay. In the present paper, we generalize this result to an arbitrary number of antennas at the relay. The following theorem is our main result.

Theorem 1: The DMT of the rotate-and-forward scheme in a two-hop MIMO relay channel with i.i.d. Rayleigh fading is given by

$$
d_{\min \{m, p\}, n}(r),
$$

where $d_{m, n}(r)$ denotes the DMT of a classical $m \times n$ MIMO channel.

According to the cut-set bound from information theory, it is readily shown that the DMT of the endto-end channel with any relaying scheme is dominated by both the DMT of the source-relay cut and the relaydestination cut, i.e. by $\min \left\{d_{m, n}(r), d_{n, p}(r)\right\}$, which coincides with (3). So what the above theorem shows is that the rotate-and-forward scheme achieves the optimal DMT in this setting. To the authors' best knowledge, this is the first distributed linear relaying scheme that is shown to achieve the optimal DMT for an arbitrary number of antennas at the source, relay and destination.

The proof of the above theorem is provided in Section IV for the case where $\min \{m, p\} \geq n$ (the general case is an easy extension, that will be treated in the journal version of this paper). We follow the procedure decribed in [17] for analyzing the DMT of the rotate-and-forward scheme, extending it to the case of an arbitrary number of antennas at the relay. The core of this extension relies on the argument exposed in the next section.

\section{Classical MiMO Channel}

In this section, we describe a new approach for obtaining a lower bound on the DMT of the classical MIMO channel. This approach turns out to be the key for proving the DMT optimality of the rotate-andforward scheme. Let us consider a classical MIMO channel with $n$ antennas at the source and $m$ antennas at the destination, and let $H_{n}$ be the $m \times n$ channel matrix, with i.i.d. circularly symmetric complex Gaussian entries with unit variance. In this case, the mutual information between the source and destination is approximated at high SNR by $I(\mathrm{SNR}) \approx \log \operatorname{det}\left(I_{n}+\right.$
SNR $H_{n}^{*} H_{n}$ ). The key proposition of this section is the following.

Proposition 1: Assume without loss of generality that $m \geq n$. The following lower bound holds:

$$
\begin{aligned}
\operatorname{det} & \left(I_{n}+\operatorname{SNR} H_{n}^{*} H_{n}\right) \\
& =\sum_{\mathcal{J} \subseteq\{1, \ldots, n\}} \operatorname{SNR}^{|\mathcal{J}|} \operatorname{det}\left(H_{n}^{*} H_{n}\right)_{\mathcal{J}} \\
\geq & 1+\operatorname{SNR} \sum_{i=1}^{n} p_{i}^{(m)}+\sum_{k=2}^{n} \mathrm{SNR}^{k} \sum_{i=1}^{n-k+1} a_{k i}^{(m)},(4)
\end{aligned}
$$

where $p_{i}^{(m)}=\left\|h_{i}^{(m)}\right\|^{2}$, with $h_{i}^{(m)} \in \mathbb{C}^{m \times 1}$ being the $i^{t h}$ column of the matrix $H_{n}$, and

$$
\begin{aligned}
a_{k i}^{(m)} & =p_{i}^{(m)}\left(\prod_{j=n-k+2}^{n} p_{j}^{(m)}\right) \\
& \times \prod_{l=1}^{k-1}\left(u_{i, n-l+1, \ldots, n}^{(m)} \prod_{j=n-k+2}^{n-l} u_{j, n-l+1, \ldots, n}^{(m)}\right)
\end{aligned}
$$

where by convention, $\prod_{j=p}^{q} c_{j}=1$ if $q<p$. The random variables $p_{i}^{(m)}$ are independent and $\operatorname{Gamma}(m, 1)$ distributed:

$$
p_{p_{i}^{(m)}}(x)=\frac{x^{m-1} e^{-x}}{\Gamma(m)} 1_{x \geq 0},
$$

while the random variables $u_{\mathcal{J}}^{(m)}$ appearing in (5) with $\mathcal{J} \subseteq\{1, \ldots, n\}$ and $|\mathcal{J}|=l+1$ are independent and $\operatorname{Beta}(m-l, 1)$ distributed:

$$
p_{u_{\mathcal{J}}^{(m)}}(u)=(m-l) u^{m-l-1} 1_{0 \leq u \leq 1} .
$$

Furthermore, the random variables $p_{i}^{(m)}$ and $u_{\mathcal{J}}^{(m)}$ are independent.

As an illustration, in the particular case $n=3$, this proposition reads

$$
\begin{aligned}
\operatorname{det}\left(I_{3}\right. & \left.+\operatorname{SNR} H_{3}^{*} H_{3}\right) \\
\geq & 1+\operatorname{SNR}\left(p_{1}^{(m)}+p_{2}^{(m)}+p_{3}^{(m)}\right) \\
& +\operatorname{SNR}^{2}\left(p_{1}^{(m)} p_{3}^{(m)} u_{13}^{(m)}+p_{2}^{(m)} p_{3}^{(m)} u_{23}^{(m)}\right) \\
& +\operatorname{SNR}^{3} p_{1}^{(m)} p_{2}^{(m)} p_{3}^{(m)} u_{13}^{(m)} u_{23}^{(m)} u_{123}^{(m)},
\end{aligned}
$$

where $p_{i}(m)$ are $\operatorname{Gamma}(m, 1)$-distributed,

$$
\begin{aligned}
& u_{13}^{(m)} \text { and } u_{23}^{(m)} \text { are } \operatorname{Beta}(m-1,1) \text {-distributed, } \\
& u_{123}^{(m)} \text { is } \operatorname{Beta}(m-2,1) \text {-distributed, }
\end{aligned}
$$

and all these random variables are independent.

The proof of the above proposition is given in the Appendix. Let us try here to provide some intuition 
on the result and also to briefly explain its main consequence. Notice first that contrary to the approach taken in [3], the expressions (4) and (5) do not involve the eigenvalues of the random matrix $H_{n}^{*} H_{n}$; instead, the random variables $p_{i}^{(m)}$ are related to the norms of the columns of $H_{n}$, while the random variables $u_{\mathcal{J}}^{(m)}$ are related to the angles between these columns.

Second, notice that the subdeterminants $\operatorname{det}\left(H_{n}^{*} H_{n}\right)_{\mathcal{J}}$ appearing in the first line of (4) are intricate random variables, from which one could hardly deduce anything on the outage probability. Instead, the random variables $a_{k i}^{(m)}$ defined in (5) have a much nicer structure. In particular, writing down explicitly what the $a_{k i}^{(m)}$ are for the first two values of $k$ :

$$
\begin{aligned}
a_{2 i}^{(m)} & =p_{i}^{(m)} p_{n}^{(m)} u_{i n}^{(m)}, \\
a_{3 i}^{(m)} & =p_{i}^{(m)} p_{n-1}^{(m)} p_{n}^{(m)} u_{i n}^{(m)} u_{n-1, n}^{(m)} u_{i, n-1, n}^{(m)},
\end{aligned}
$$

we discover the inherent hierarchy of the lower bound (4): each random variable $a_{k i}^{(m)}$ is the product of $a_{k-1, i}^{(m)}$ and some other random variables independent of $a_{k-1, i}^{(m)}$. Moreover, the final expression in (4) is a simple function of independent variables, from which the diversity order can be easily deduced, using the methodology of [3]. Without entering into the proof details, we obtain the following main outage events and diversity orders, depending on the multiplexing gain $r$ :

- if $r=0$, then the main outage event occurs when the $n$ random variables $\left(p_{i}^{(m)}, i=1, \ldots, n\right)$ are of order $\mathrm{SNR}^{-1}$, which leads to a diversity order $d(0)=n m$.

- if $r=k \in\{1, \ldots, n\}$, then the main outage event occurs when the $n-k$ random variables $\left(u_{i, n-k+1, \ldots, n}^{(m)}, i=1, \ldots, n-k\right)$ are of order $\mathrm{SNR}^{-1}$, which leads to a diversity order $d(k)=(n-k)(m-k)$.

\section{ROTATE-AND-ForwARD SCHEME AND DMT ANALYSIS}

In this section, we provide the main steps for the proof of Theorem 1, restricting ourselves to the case where $\min \{m, p\} \geq n$ for simplicity. The first steps of this proof, which have already been presented in [17], will be exposed without detailed proof. (Detailed proofs will be stated in the journal version of this paper.) In order to obtain a lower bound on the diversity order, we look for an asymptotic lower bound on the mutual information $I_{T}(\mathrm{SNR})$ given by (2). It can first be shown that

$$
I_{T}(\mathrm{SNR}) \geq\left(\frac{L-1}{L}\right)^{n-1} I^{\star}(\mathrm{SNR})-2,
$$

where we recall that $T=L^{n}$, and $I^{\star}(\mathrm{SNR})$ is defined as

$$
I^{\star}(\mathrm{SNR}) \triangleq \log \left(\mathbb{E}_{\theta} \operatorname{det}\left(I_{n}+\operatorname{SNR} R_{\theta} F F^{*} R_{\theta}^{*} G^{*} G\right)\right) .
$$

Here, $R_{\theta}=\operatorname{diag}\left(\exp \left(i \theta_{1}\right), \ldots, \exp \left(i \theta_{n}\right)\right)$ and $\mathbb{E}_{\theta}$ denotes the expectation over the random variables $\theta_{1}, \ldots, \theta_{n}$, which are assumed to be independent and uniformly distributed on $[0,2 \pi)$. From this, we deduce that the diversity order corresponding to $I_{T}(\mathrm{SNR})$ is approached by that corresponding to $I^{\star}(\mathrm{SNR})$ as $L \rightarrow$ $\infty$. Next, it can be shown that

$I_{T}(\mathrm{SNR}) \leq \log \left(\mathbb{E}_{\theta}\left(\operatorname{det}\left(I_{n}+\mathrm{SNR} R_{\theta} F F^{*} R_{\theta}^{*} G^{*} G\right)\right)\right)+n$, where the explicit computation of the expectation on the right-hand side gives

$$
\begin{aligned}
& \mathbb{E}_{\theta}\left(\operatorname{det}\left(I_{n}+\operatorname{SNR} R_{\theta} F F^{*} R_{\theta}^{*} G^{*} G\right)\right) \\
& =\sum_{\mathcal{J} \subseteq\{1, \ldots, n\}} \operatorname{SNR}^{|\mathcal{J}|} \operatorname{det}\left(F F^{*}\right)_{\mathcal{J}} \operatorname{det}\left(G^{*} G\right)_{\mathcal{J}}(8)
\end{aligned}
$$

Adopting now the methodology of the previous section, the following lower bound on the last expression can be obtained:

$$
\begin{aligned}
\sum_{\mathcal{J} \subseteq\{1, \ldots, n\}} \operatorname{SNR}^{|\mathcal{J}|} \operatorname{det}\left(F F^{*}\right)_{\mathcal{J}} \operatorname{det}\left(G^{*} G\right)_{\mathcal{J}} \\
\geq 1+\operatorname{SNR} \sum_{i=1}^{n} p_{i}^{(m)} q_{i}^{(p)} \\
+\sum_{k=2}^{n} \operatorname{SNR}^{k} \sum_{i=1}^{n-k+1} a_{k i}^{(m)} b_{k i}^{(p)},
\end{aligned}
$$

where $a_{k i}^{(m)}$ is given by (5) and $b_{k i}^{(p)}$ is given by a similar expression, with the random variables $p_{i}^{(m)}$ replaced by $q_{i}^{(p)}$ and the $u_{\mathcal{J}}^{(m)}$ replaced by $v_{\mathcal{J}}^{(p)}$. Notice also that here, $p_{i}^{(m)}=\left\|f_{i}^{(m)}\right\|^{2}$ and $q_{i}^{(p)}=\left\|g_{i}^{(p)}\right\|^{2}$, where $f_{i}^{(m)}$ denotes the $i^{\text {th }}$ row of $F$ and $g_{i}^{(p)}$ denotes the $i^{\text {th }}$ column of $G$, respectively. All the random variables $p_{i}^{(m)}, q_{i}^{(p)}, u_{\mathcal{J}}^{(m)}$ and $v_{\mathcal{J}}^{(p)}$ appearing above are independent and distributed as

$$
\begin{aligned}
& p_{p_{i}^{(m)}}(x)=\frac{x^{m-1} e^{-x}}{\Gamma(m)} 1_{x \geq 0}, \\
& p_{q_{i}^{(p)}}(y)=\frac{y^{p-1} e^{-y}}{\Gamma(p)} 1_{y \geq 0}, \\
& p_{u_{\mathcal{J}}^{(m)}}(u)=(m-l) u^{m-l-1} 1_{0 \leq u \leq 1}, \\
& p_{v_{\mathcal{J}}^{(p)}}(v)=(p-l) v^{p-l-1} 1_{0 \leq v \leq 1} .
\end{aligned}
$$


for $|\mathcal{J}|=l+1$. Gathering all pieces together, we obtain

$$
\begin{aligned}
& \mathbb{P}_{\text {out }}(r \log \mathrm{SNR}) \\
& \dot{\mathbb{P}}\left(1+\mathrm{SNR} \sum_{i=1}^{n} p_{i}^{(m)} q_{i}^{(p)}\right. \\
& \left.\quad+\sum_{k=2}^{n} \mathrm{SNR}^{k} \sum_{i=1}^{n-k+1} a_{k i}^{(m)} b_{k i}^{(p)}<r \log \mathrm{SNR}\right) .
\end{aligned}
$$

Operating the following change of variables:

$$
\begin{aligned}
& p_{i}^{(m)}=\mathrm{SNR}^{-\alpha_{i}}, \quad q_{i}^{(p)}=\mathrm{SNR}^{-\beta_{i}}, \\
& u_{\mathcal{J}}^{(m)}=\mathrm{SNR}^{-\gamma_{\mathcal{J}}}, \quad v_{\mathcal{J}}^{(p)}=\mathrm{SNR}^{-\delta_{\mathcal{J}}} .
\end{aligned}
$$

and applying Laplace's integration method from [3] in order to compute the diversity order, we obtain the following lower bound on the diversity:

$$
\begin{aligned}
d(r) \geq & d_{L B}(r)=\min \left\{m \sum_{i=1}^{n} \alpha_{i}+p \sum_{i=1}^{n} \beta_{i}\right. \\
& +\sum_{k=1}^{n-1}(m-k) \sum_{i=1}^{n-k} \gamma_{i, n-k+1, \cdots, n} \\
& \left.+\sum_{k=1}^{n-1}(p-k) \sum_{i=1}^{n-k} \delta_{i, n-k+1, \cdots, n}\right\},
\end{aligned}
$$

subject to $\alpha_{i} \geq 0, \beta_{i} \geq 0, \gamma_{\mathcal{J}} \geq 0, \delta_{\mathcal{J}} \geq 0$ and

$$
\begin{gathered}
\max \left\{0,\left\{1-\alpha_{i}\right\}_{i=1}^{n},\left\{2-\alpha_{i}-\alpha_{n}-\gamma_{i n}\right\}_{i=1}^{n-1}, \cdots,\right. \\
n-\sum_{i=1}^{n} \alpha_{i}-\sum_{i=1}^{n-1} \gamma_{i n}-\cdots-\gamma_{1,2, \cdots, n}, \\
\left\{1-\beta_{i}\right\}_{i=1}^{n},\left\{2-\beta_{i}-\beta_{n}-\delta_{i n}\right\}_{i=1}^{n-1}, \cdots, \\
\left.n-\sum_{i=1}^{n} \beta_{i}-\sum_{i=1}^{n-1} \delta_{i n}-\cdots-\delta_{1,2, \cdots, n}\right\} \leq r .
\end{gathered}
$$

We now separate the analysis into two cases: $m=p$ and $m \neq p$.

\section{A. Case $m=p$}

Due to the symmetry of the problem, the optimal solution is such that $\alpha_{i}=\beta_{j}$ for all $1 \leq i, j \leq n$ and $\gamma_{\mathcal{I}}=\delta_{\mathcal{J}}$ for all $\mathcal{I}, \mathcal{J}$ such that $|\mathcal{I}|=|\mathcal{J}|$. For simplicity, suppose first that $r=k$ for $k \in$ $\{0,1,2, \cdots, n\}$. To satisfy the above constraint, all the variables which have less than $k+1$ indices must be equal to 0 . Furthermore, the variables with $k+1$ indices should be set to 1 in order to satisfy the constraint. This in turn enforces that all the other variables with more than $k+1$ indices are equal to 0 . As a result, $d(k)=(m-k)(n-k)$ is obtained.
In general, the solution of this optimization problem is the following. For $k-1 \leq r \leq k$ and $k \in\{1, \cdots, n\}$, we have

$$
\begin{aligned}
\alpha_{i}=\beta_{i} & =(1-r)^{+}, \\
\delta_{\mathcal{J}}=\gamma_{\mathcal{J}} & = \begin{cases}k-r, & \text { if }|\mathcal{J}|=k . \\
r-k+1, & \text { if }|\mathcal{J}|=k+1, \\
0, & \text { otherwise. }\end{cases}
\end{aligned}
$$

Therefore, the lower bound on the DMT of the rotateand-forward scheme is given by

$$
\begin{aligned}
d_{L B}(r)= & (n-k+1)(m-k+1)(k-r) \\
& +(n-k)(m-k)(r-k+1)=d_{m, n}(r),
\end{aligned}
$$

which matches the cutset upper bound.

\section{B. Case $m \neq p$}

Let $q$ denote the minimum of $m$ and $p$. By the cutset bound, the diversity of the system can be upperbounded by the diversity of each stage, i.e.

$$
d(r) \leq \min \left\{d_{m, n}(r), d_{n, p}(r)\right\}=d_{n, q}(r) .
$$

- If $m>p=q$, we can simply not send any signal in $m-p$ antennas at the source node. So we can apply the result giving us a lower bound on the DMT which matches the upper bound in (10). Therefore, $d(r)=$ $d_{n, q}(r)$.

- If $q=m<p$, we can simply ignore the received signal in $p-m$ antennas at the destination node. Again, using the cutset bound, we deduce that $d(r)=d_{n, q}(r)$.

This completes the proof of Theorem 1.

\section{Conclusion And Perspectives}

In this paper, we have proved that the rotate-andforward scheme presented in [17] is DMT optimal for an arbitrary number of antennas at the relay. This problem was open up to now, and the DMT optimality of the scheme was only shown for the case of two antennas at the relay. The new result shows that the diversity-multiplexing tradeoff for a two-hop relay channel can be achieved with a quite practical linear relaying scheme. The technique that we used for computing the DMT was to set up a lower bound for the determinant of a random matrix with an expression which is a simple function of independent random variables with known distributions. Regarding future work, the optimality of the rotate-and-forward scheme may hold in more generality, in a multi-hop relay network, with arbitrary number of antennas at the relays and arbitrary number of relays. However, the proof of this fact remains open. 
APPENDIX

\section{A. Proof of Proposition 1}

We prove the proposition by induction. First, it is obvious that for any $m \geq n=1, \operatorname{det}\left(I_{n}+\operatorname{SNR} H_{n}^{*} H_{n}\right)=$ $1+\mathrm{SNR}\left\|h_{1}^{(m)}\right\|^{2}$ satisfies (4). Now, let us suppose that equation (4) holds for some $m \geq n$; we will show that it remains true for $m+1$ and $n+1$, proving therefore Proposition 1 for all possible values of $m \geq n$.

As the matrix $H_{n+1}$ is an $(m+1) \times(n+1)$ matrix with i.i.d. circularly symmetric Gaussian entries, it can be expressed as

$$
H_{n+1}=U \tilde{H}_{n+1},
$$

where $U$ is uniformly distributed on the set of $(m+$ $1) \times(m+1)$ unitary matrices (Haar distribution) and $\tilde{H}_{n+1}$ is given by

$\tilde{H}_{n+1}$

$=\left[\tilde{h}_{1}^{(m+1)}, \tilde{h}_{2}^{(m+1)}, \cdots, \tilde{h}_{n}^{(m+1)},\left\|h_{n+1}{ }^{(m+1)}\right\| e_{m+1}\right]$,

where $e_{m+1}=[0, \cdots, 0,1]^{*} \in \mathbb{C}^{(m+1) \times 1}$ and ${\tilde{h_{i}}}^{(m+1)} \in \mathbb{C}^{(m+1) \times 1}$ are independent column vectors with i.i.d. circularly symmetric Gaussian entries. Hence,

$$
\begin{aligned}
& \operatorname{det}\left(I_{n+1}+\operatorname{SNR} H_{n+1}^{*} H_{n+1}\right) \\
& =\operatorname{det}\left(I_{n+1}+\operatorname{SNR} \tilde{H}_{n+1}^{*} U^{*} U \tilde{H}_{n+1}\right) \\
& =\operatorname{det}\left(I_{n+1}+\operatorname{SNR} \tilde{H}_{n+1}^{*} \tilde{H}_{n+1}\right) .
\end{aligned}
$$

Define now $\tilde{H}_{n}$ the $m \times n$ reduced matrix of $\tilde{H}_{n+1}$ without the $(m+1)^{t h}$ row and $(n+1)^{t h}$ column.

Lemma 1: Let $\mathcal{I} \subseteq\{1,2, \cdots, n\}$ and $\mathcal{J}=\mathcal{I} \cup$ $\{n+1\}$. Let also $\left(\tilde{H}_{n+1}^{*} \tilde{H}_{n+1}\right) \mathcal{J}$ denote the matrix constructed by rows and columns of the matrix $\tilde{H}_{n+1}^{*} \tilde{H}_{n+1}$ for which the indices are in $\mathcal{J}$. Then the determinant of $\left(\tilde{H}_{n+1}^{*} \tilde{H}_{n+1}\right)_{\mathcal{J}}$ can be expressed as

$$
\operatorname{det}\left(\tilde{H}_{n+1}^{*} \tilde{H}_{n+1}\right)_{\mathcal{J}}=\left\|\tilde{h}_{n+1}^{(m+1)}\right\|^{2} \operatorname{det}\left(\tilde{H}_{n}^{*} \tilde{H}_{n}\right)_{\mathcal{I}} .
$$

Proof: The matrix $\left(\tilde{H}_{n+1}^{*} \tilde{H}_{n+1}\right)_{\mathcal{J}}$ can be expressed as

$$
\left(\tilde{H}_{n+1}^{*} \tilde{H}_{n+1}\right)_{\mathcal{J}}=\left(\begin{array}{cc}
A & b \\
b^{*} & c
\end{array}\right)
$$

where $A \in \mathbb{C}^{|\mathcal{I}| \times|\mathcal{I}|}, b \in \mathbb{C}^{|\mathcal{I}| \times 1}$ and $c \in \mathbb{C}$ are given by

$$
\begin{aligned}
A & =\left\{\sum_{i=1}^{m+1} \overline{\tilde{h}_{j, i}^{(m+1)}} \tilde{h}_{k, i}^{(m+1)}\right\}_{j, k \in \mathcal{I}}, \\
b & =\left\{\left\|h_{n+1}^{(m+1)}\right\| \overline{\tilde{h}_{j, m+1}^{(m+1)}}\right\}_{j \in \mathcal{I}}, \\
c & =\left\|h_{n+1}^{(m+1)}\right\|^{2},
\end{aligned}
$$

where $\tilde{h}_{k, i}^{(m+1)}$ is the $i^{t h}$ entry of column vector $\tilde{h}_{k}^{(m+1)}$. We know that

$$
\operatorname{det}\left(\begin{array}{cc}
A & b \\
b^{*} & c
\end{array}\right)=c \operatorname{det}\left(A-\frac{1}{c} b b^{*}\right)
$$

and therefore obtain

$$
\begin{aligned}
& \operatorname{det}\left(\tilde{H}_{n+1}^{*} \tilde{H}_{n+1}\right)_{\mathcal{J}} \\
& =\left\|h_{n+1}^{(m+1)}\right\|^{2} \operatorname{det}\left\{\sum_{i=1}^{m} \overline{\tilde{h}_{j, i}^{(m+1)}} \tilde{h}_{k, i}^{(m+1)}\right\}_{j, k \in \mathcal{I}} \\
& =\left\|h_{n+1}^{(m+1)}\right\|^{2} \operatorname{det}\left(\tilde{H}_{n}^{*} \tilde{H}_{n}\right)_{\mathcal{I}} .
\end{aligned}
$$

This completes the proof of Lemma 1 .

Lemma 2: The following inequality holds:

$$
\begin{aligned}
& \operatorname{det}\left(I_{n+1}+\operatorname{SNR} \tilde{H}_{n+1}^{*} \tilde{H}_{n+1}\right) \\
& \geq 1+\operatorname{SNR} \sum_{i=1}^{n}\left\|\tilde{h}_{i}^{(m+1)}\right\|^{2} \\
& \quad+\operatorname{SNR}\left\|\tilde{h}_{n+1}^{(m+1)}\right\|^{2} \operatorname{det}\left(I_{n}+\operatorname{SNR} \tilde{H}_{n}^{*} \tilde{H}_{n}\right) .
\end{aligned}
$$

Proof: The proof is done in two steps:

$$
\begin{aligned}
\operatorname{det}\left(I_{n+1}+\operatorname{SNR} \tilde{H}_{n+1}^{*} \tilde{H}_{n+1}\right) & \sum_{\mathcal{J} \subseteq\{1,2, \cdots, n+1\}} \operatorname{SNR}^{|\mathcal{J}|} \operatorname{det}\left(\tilde{H}_{n+1}^{*} \tilde{H}_{n+1}\right)_{\mathcal{J}} \\
\geq & 1+\operatorname{SNR} \sum_{i=1}^{n}\left\|\tilde{h}_{i}^{(m+1)}\right\|^{2} \\
& +\sum_{\substack{\mathcal{J}=\mathcal{I} \cup\{n+1\} \\
\mathcal{I} \subseteq\{1,2, \cdots, n\}}} \operatorname{SNR}^{|\mathcal{J}|} \operatorname{det}\left(\tilde{H}_{n+1}^{*} \tilde{H}_{n+1}\right)_{\mathcal{J}} .
\end{aligned}
$$

By Lemma 1, we further obtain

$$
\begin{aligned}
& \sum_{\substack{\mathcal{J}=\mathcal{I} \cup\{n+1\} \\
\mathcal{I} \subseteq\{1,2, \cdots, n\}}} \operatorname{SNR}^{|\mathcal{J}|} \operatorname{det}\left(\tilde{H}_{n+1}^{*} \tilde{H}_{n+1}\right)_{\mathcal{J}} \\
& =\operatorname{SNR}\left\|\tilde{h}_{n+1}^{(m+1)}\right\|^{2} \sum_{\mathcal{I} \subseteq\{1,2, \cdots, n\}} \operatorname{SNR}^{|\mathcal{I}|} \operatorname{det}\left(\tilde{H}_{n}^{*} \tilde{H}_{n}\right)_{\mathcal{I}} \\
& =\operatorname{SNR}\left\|\tilde{h}_{n+1}^{(m+1)}\right\|^{2} \operatorname{det}\left(I_{n}+\operatorname{SNR} \tilde{H}_{n}^{*} \tilde{H}_{n}\right),
\end{aligned}
$$

so the proof of Lemma 2 is complete. 
Lemma 3: If $X$ and $Y$ are two independent Gammadistributed random variables with parameters $(p, 1)$ and $(q, 1)$ respectively, then the random variable $Z=\frac{X}{X+Y}$ is independent of both $X$ and $Y$ and is distributed according to the Beta distribution with parameters $(p, q)$, i.e.

$$
\begin{aligned}
& p_{X}(x)=\frac{x^{p-1} e^{-x}}{\Gamma(p)} 1_{x \geq 0}, \quad p_{Y}(y)=\frac{y^{q-1} e^{-y}}{\Gamma(q)} 1_{y \geq 0}, \\
& p_{Z}(z)=\frac{z^{p-1}(1-z)^{q-1}}{\int_{0}^{1} u^{p-1}(1-u)^{q-1} d u} 1_{0 \leq z \leq 1} .
\end{aligned}
$$

Proof: See [19].

Lemma 4: The random variables $a_{k i}^{(m)}$ defined in (5) satisfy the following recursive relation:

$$
a_{k+1, i}^{(m+1)}=p_{n+1}^{(m+1)} a_{k i}^{(m)} .
$$

Proof: Remembering definition (5):

$$
\begin{aligned}
a_{k i}^{(m)} & =p_{i}^{(m)}\left(\prod_{j=n-k+2}^{n} p_{j}^{(m)}\right) \\
& \times \prod_{l=1}^{k-1}\left(u_{i, n-l+1, \ldots, n}^{(m)} \prod_{j=n-k+2}^{n-l} u_{j, n-l+1, \ldots, n}^{(m)}\right)
\end{aligned}
$$

and defining further

$$
u_{j, n+1}^{(m+1)}=\frac{p_{j}^{(m)}}{p_{j}^{(m+1)}}, \quad j=1, \ldots, n,
$$

and

$u_{j, n-l+1, \ldots, n+1}^{(m+1)}=u_{j, n-l+1, \ldots, n}^{(m)}, \quad j=1, \ldots, n-l, l \geq 1$,

we obtain

$$
\begin{aligned}
& p_{n+1}^{(m+1)} a_{k i}^{(m)}=p_{i}^{(m+1)}\left(\prod_{j=n-k+2}^{n+1} p_{j}^{(m+1)}\right) \\
& \quad \times u_{i, n+1}^{(m+1)}\left(\prod_{j=n-k+2}^{n} u_{j, n+1}^{(m+1)}\right) \\
& \quad \times \prod_{l=1}^{k-1}\left(u_{i, n-l+1, \ldots, n+1}^{(m+1)} \prod_{j=n-k+2}^{n-l} u_{j, n-l+1, \ldots, n+1}^{(m+1)}\right) \\
& \quad=a_{k+1, i}^{(m+1)} .
\end{aligned}
$$

Let us check that the random variables appearing in this expression are distributed according to what is stated in Proposition 1. Indeed, by definition, $p_{j}^{(m+1)}=\left\|h_{j}^{(m+1)}\right\|^{2}$ are $\operatorname{Gamma}(m+1,1)$ distributed, $u_{j, n-l+1, \ldots, n+1}^{(m+1)}$ are $\operatorname{Beta}(m-l, 1)=\operatorname{Beta}((m+1)-$ $(l+1), 1)$ distributed for $l \geq 1$, and all these variables are independent. What remains therefore to be checked is that the new random variables $u_{j, n+1}^{(m+1)}$ defined above are $\operatorname{Beta}(m+1,1)$ distributed and also independent of all the other random variables:

$u_{j, n+1}^{(m+1)}=\frac{p_{j}^{(m)}}{p_{j}^{(m+1)}}=\frac{\left\|h_{j}^{(m)}\right\|^{2}}{\left\|h_{j}^{(m+1)}\right\|^{2}}=\frac{\left\|h_{j}^{(m)}\right\|^{2}}{\left\|h_{j}^{(m)}\right\|^{2}+\left|h_{j, m+1}^{(m+1)}\right|^{2}}$

where $h_{j, m+1}^{(m+1)}$ is the $(m+1)^{t h}$ entry of the column vector $h_{j}^{(m+1)}$. Therefore, by Lemma 3, the new random variables $u_{j, n+1}^{(m+1)}$ are $\operatorname{Beta}(m+1,1)$ distributed and independent of the random variables $p_{j}^{(m+1)}$. Furthermore, these new random variables are functions of $\left\|h_{j}^{(m)}\right\|^{2}$ and $\left|h_{j, m+1}^{(m+1)}\right|^{2}$, which are independent of all the random variables $u_{j, n-l+1, \ldots, n+1}^{(m+1)}=u_{j, n-l+1, \ldots, n}^{(m)}$, $l \geq 1$.

We are now ready to complete the proof of Proposition 1. By Lemma 2,

$$
\begin{aligned}
& \operatorname{det}\left(I_{n+1}+\operatorname{SNR} H_{n+1}^{*} H_{n+1}\right) \\
& =\operatorname{det}\left(I_{n+1}+\operatorname{SNR} \tilde{H}_{n+1}^{*} \tilde{H}_{n+1}\right) \\
& \geq 1+\operatorname{SNR} \sum_{i=1}^{n}\left\|\tilde{h}_{i}^{(m+1)}\right\|^{2} \\
& \quad+\operatorname{SNR}\left\|\tilde{h}_{n+1}^{(m+1)}\right\|^{2} \operatorname{det}\left(I_{n}+\operatorname{SNR} \tilde{H}_{n}^{*} \tilde{H}_{n}\right),
\end{aligned}
$$

and by the induction assumption, Proposition 1 holds for $\operatorname{det}\left(I_{n}+\operatorname{SNR} \tilde{H}_{n}^{*} \tilde{H}_{n}\right)$, as the matrix $\tilde{H}_{n}$ has i.i.d. circularly symmetric Gaussian entries with unit variance. Therefore,

$$
\begin{aligned}
& \operatorname{det}\left(I_{n+1}+\mathrm{SNR} H_{n+1}^{*} H_{n+1}\right) \\
& \geq 1+\mathrm{SNR} \sum_{i=1}^{n}\left\|\tilde{h}_{i}^{(m+1)}\right\|^{2}+\mathrm{SNR}\left\|\tilde{h}_{n+1}^{(m+1)}\right\|^{2} \\
&\left(1+\mathrm{SNR} \sum_{i=1}^{n} p_{i}^{(m)}+\sum_{k=2}^{n} \mathrm{SNR}^{k} \sum_{i=1}^{n-k+1} a_{k i}^{(m)}\right) \\
&= 1+\mathrm{SNR} \sum_{i=1}^{n} p_{i}^{(m+1)}+\mathrm{SNR} p_{n+1}^{(m+1)} \\
& \times\left(1+\mathrm{SNR} \sum_{i=1}^{n} p_{i}^{(m)}+\sum_{k=2}^{n} \mathrm{SNR}^{k} \sum_{i=1}^{n-k+1} a_{k i}^{(m)}\right)
\end{aligned}
$$

as $\left\|\tilde{h}_{i}^{(m+1)}\right\|^{2}=\left\|h_{i}^{(m+1)}\right\|^{2}=p_{i}^{(m+1)}$. We finally 
obtain

$$
\begin{aligned}
& \operatorname{det}\left(I_{n+1}+\mathrm{SNR} H_{n+1}^{*} H_{n+1}\right) \\
& \geq 1+\mathrm{SNR} \sum_{i=1}^{n+1} p_{i}^{(m+1)} \\
& +\mathrm{SNR}^{2} \sum_{i=1}^{n} p_{i}^{(m+1)} p_{n+1}^{(m+1)} u_{i, n+1}^{(m+1)} \\
& \quad+\sum_{k=2}^{n} \mathrm{SNR}^{k+1} \sum_{i=1}^{n-k+1} p_{n+1}^{(m+1)} a_{k i}^{(m+1)} \\
& =1+\mathrm{SNR}_{i=1}^{n+1} p_{i}^{(m+1)} \\
& \quad+\sum_{k=2}^{n+1} \mathrm{SNR}^{k} \sum_{i=1}^{(n+1)-k+1} a_{k i}^{(m+1)},
\end{aligned}
$$

using the fact that $a_{2 i}^{(m+1)}=p_{i}^{(m+1)} p_{n+1}^{(m+1)} u_{i, n+1}^{(m+1)}$, as well as Lemma 4. This completes the proof of Proposition 1.

\section{REFERENCES}

[1] G. J. Foschini and M. J. Gans, "On Limits of Wireless Communications in a Fading Environment when Using Multiple Antennas", Wireless Personal Communications, vol. 6, no. 3, March 1998, pp. 311-335.

[2] E. Telatar, "Capacity of Multi-antenna Gaussian Channels", European Trans. on Telecommunications, vol. 10, no. 6, June 1999, pp. 585-596.

[3] L. Zheng and D. Tse, "Diversity and Multiplexing: A Fundamental Tradeoff in Multiple Antenna Channels", IEEE Trans. Inf. Theory, vol. 49, no. 5, pp. 1073-96, May 2003.

[4] S. Yang, J.-C. Belfiore, and G. Rekaya, "Perfect space-time block codes for parallel MIMO channels", in Proc. 2006 IEEE International Symposium on Information Theory, Seattles, Jul. 2006.

[5] J. N. Laneman and G. W. Wornell, "Distributed space-timecoded protocols for exploiting cooperative diversity in wireless networks," IEEE Trans. Inf. Theory, vol. 49, no. 10, pp. 24152425, Oct. 2003.

[6] J. N. Laneman, D. N. C. Tse, and G. W. Wornell, "Cooperative diversity in wireless networks: Efficient protocols and outage behavior," IEEE Trans. Inf. Theory, vol. 50, no. 12, pp. 30623080, Dec. 2004.

[7] T. Hunter, S. Sanayei, and A. Nosratinia, "Outage analysis of coded cooperation," IEEE Trans. Inf. Theory, vol. 52, no. 2, pp. 375-391, Feb. 2006.

[8] R. U. Nabar, H. Bölcskei, and F. W. Kneubühler, "Fading relay channels: Performance limits and space-time signal design," IEEE J. Sel. Areas Commun., vol. 22, no. 6, pp. 1099-1109, Aug. 2004.

[9] K. Azarian, H. El Gamal, and P. Schniter, "On the achievable diversity-multiplexing tradeoff in half-duplex cooperative channels," IEEE Trans. Inf. Theory, vol. 51, no. 12, pp. 41524172, Dec. 2005.

[10] S. Yang and J.-C. Belfiore, "Towards the optimal amplifyand-forward cooperative diversity scheme," IEEE Trans. Inf. Theory, vol. 53, no. 9, pp. 3114-3126, Sep. 2007.
[11] M. Yuksel and E. Erkip, "Multi-antenna cooperative wireless systems: A diversity multiplexing tradeoff perspective," IEEE Trans. Inf. Theory, vol. 53, no. 10, pp. 3371-3393, Oct. 2007.

[12] Y. Jing and B. Hassibi, "Distributed space-time coding in wireless relay networks," IEEE Trans. Wireless Commun., vol. 5, no. 12, pp. 3524-3536, Dec. 2006.

[13] P. Elia, K. Vinodh, M. Anand, and P. V. Kumar, "D-MG tradeoff and optimal codes for a class of af and df cooperative communication protocols," IEEE Trans. Inf. Theory, vol. 55, no. 7, pp. 3161-3185, Jul. 2009.

[14] S. Avestimehr, S. Diggavi, and D. N. C. Tse, "Wireless network information flow: a deterministic approach," IEEE Trans. Inf. Theory, June 2009, submitted. [Online]. Available: http://arxiv.org/abs/0906.5394

[15] D. Gündüz, M. A. Khojastepour, A. Goldsmith and H. V. Poor. "Multi-hop MIMO relay networks: diversity-multiplexing trade-off analysis," IEEE Trans. Wireless Commun., vol. 9, no.10, pp. 1738-1747, May 2010.

[16] S. Yang, J.-C. Belfiore, "Diversity of MIMO multi-hop relay channels", Aug. 2007. [Online]. Available: http: / / arxiv. org/abs/0708.0386.

[17] S. Yang, J.-C. Belfiore, "Distributed rotation recovers spatial diversity," in Proc. 2010 IEEE International Symposium on Information Theory, Austin, Texas, June 2010.

[18] R. Pedarsani, O. Lévêque, S. Yang, "Flip-and-forward achieves the optimal diversity-multiplexing tradeoff for the two-hop MIMO relay channel, with two antennas at the relay," in Proc. Fifth International Conference on Cognitive Radio Oriented Wireless Networks and Communications (CROWNCOM), Cannes, France, June 2010.

[19] R. V. Hogg, A. T. Craig, "Introduction to mathematical statistics", Macmillan, New York, 1978. 